\title{
La presencia de rol cívico, de servicio y de infoentretenimiento en las noticias de cultura y espectáculos del periodismo chileno ${ }^{1}$
}

\section{Lía Alvear ${ }^{2}$ Claudia Mellado ${ }^{3}$}

Recibido: 2016-12-03

Enviado a pares: 2017-01-11
Aprobado por pares: 2017-05-05

Aceptado: 2017-06-01

DOI: 10.5294/pacla.2018.21.2.4

Para citar este artículo / to reference this article / para citar este artigo

Alvear, L. y Mellado, C. (2018). La presencia de rol cívico, de servicio y de infoentretenimiento en las noticias de cultura y espectáculos del periodismo chileno. Palabra Clave, 21(2), 338-363. DOI: 10.5294/pacla.2018.21.2.4

\section{Resumen}

Por medio de un análisis de contenido de noticias sobre cultura y espectáculos en los noticieros de televisión, diarios impresos, diarios digitales y noticieros de radio en Chile con mayores niveles de audiencia o lectoría, este artículo compara la presencia del rol cívico, de servicio y de infoentretenimiento en ambos frentes informativos entre las distintas plataformas analizadas. Los resultados del estudio muestran que el infoentretenimiento es un rol predominante y transversal a la cultura y el espectáculo, sin observarse diferencias significativas entre ambos frentes informativos. Por su parte, el rol de servicio está altamente presente en la cobertura cultural, a

\footnotetext{
1 Este estudio fue financiado por el proyecto de investigación Fondecyt Regular1150153 "Roles profesionales y su materialización en el contenido noticioso del periodismo chileno: un estudio comparado de la televisión, prensa online, prensa escrita, radio y redes sociales".

2 orcid.org/0000-0003-2535-8087. Centro Cultural Palacio La Moneda, Chile. liaalvear@centroculturallamoneda.cl

3 orcid.org/0000-0002-9281-1526. Pontificia Universidad Católica de Valparaíso, Chile. claudia.mellado@pucv.cl
} 
la par que el infoentretenimiento, mientras que el rol cívico no es prioridad en las noticias de ninguno de estos frentes, aunque comparativamente, su presencia es significativamente mayor en las noticias de televisión. Al considerar la influencia que el periodismo y los medios tienen en la construcción de realidad a la hora de generar, organizar y jerarquizar el contenido noticioso, estos resultados tienden a sustentar la idea de que los medios y el periodismo estarían supeditados a la generación de entretención y de necesidades de consumo de las personas más que a la creación de una identidad cultural y ciudadana.

\section{Palabras clave}

Periodismo; multiplataforma; noticias; roles profesionales; análisis de contenido; Chile; audiencia (Fuente: Tesauro de la Unesco). 


\section{The Presence of Civic, Service and Infotainment Roles in the News about Culture and Show Business of Chilean Journalism}

\section{Abstract}

Through an analysis of news content about culture and shows in television news, print newspapers, digital newspapers and radio newscasts in Chile with higher audience or readership levels, this paper compares the presence of civic, service and infotainment roles in both information fronts between the different analyzed platforms. The results of the study show that infotainment is a predominant and transversal role in culture and show business, and no significant differences are observed between the two information fronts. The service and infotainment roles, for their part, are highly present in cultural coverage, while the civic role is not a news priority for any of these fronts, although comparatively, its presence is significantly higher in television news. When considering the influence of journalism and the media on the construction of reality when generating, organizing and hierarchizing news content, these results tend to support the idea that the media and journalism would be subject to the generation of entertainment and of the consumption needs of people rather than the creation of a cultural and citizen identity.

\section{Keywords}

Journalism; multiplatform; news; professional roles; content analysis; Chile; audience (Source: Unesco Thesaurus). 


\section{A presença de papel cívico, de serviço e de info entretenimento nas notícias de cultura e espetáculos do jornalismo chileno}

\section{Resumo}

Através de uma análise de conteúdo de notícias sobre cultura e espetáculos nos noticiários de televisão, diários impressos, diários digitais e noticiários de rádio no Chile com maiores níveis de audiência ou de público leitor, este artigo compara a presença do papel cívico, de serviço e de info entretenimento em ambas as frentes de informação entre as diferentes plataformas analisadas. Os resultados do estudo mostram que o info entretenimento é um papel predominante e transversal no que refere à cultura e o espetáculo, sem observar diferenças significativas entre ambos os frentes de informação. Por outro lado, o papel de serviço e o papel do info entretenimento estão altamente presentes na cobertura cultural, enquanto que o papel cívico não é prioridade nas notícias de nenhuma destas frentes, ainda que comparativamente, sua presença é significativamente maior nas notícias de televisão. Ao considerar a influência que o jornalismo e a mídia têm na construção de realidade na hora de gerar, organizar e hierarquizar o conteúdo das notícias, estes resultados tendem a reafirmar a ideia de que a mídia e o jornalismo estariam sujeitos à geração de entretenimento e de necessidades de consumo das pessoas mais do que à criação de uma identidade cultural e cidadã.

\section{Palavras-chave}

Jornalismo; multiplataforma; notícias; papel profissional; análise de conteúdo; Chile; audiência (Fonte: Tesauro da Unesco). 


\section{Introducción}

Las investigaciones recientes dentro de los estudios sobre roles profesionales han abordado de forma conceptual y empírica la brecha que existe entre la concepción que los periodistas poseen sobre sus roles profesionales y los roles que realmente desempeñan (Vos, 2002, 2005; Tandoc, Hellmueller y Vos, 2013; Mellado y Van Dalen, 2014; Mellado, 2015; Mellado, Hellmueller y Donsbach, 2017).

Y mientras diversos investigadores han reconocido las limitaciones que la aplicación de encuestas a los periodistas tienen a la hora de pretender conocer las prácticas periodísticas (Mellado, 2015; Mellado y Lagos, 2014; Tandoc, Hellmueller y Vos, 2013; Vos, 2002), estudios más recientes — testeados y validados en diferentes regiones y paíseshan desarrollado una conceptualización y metodología consensuada sobre el abordaje de las culturas periodísticas a través del análisis de la puesta en práctica de los roles profesionales, específicamente, en el producto informativo que llega al público (Mellado y Van Dalen, 2017; Mellado, 2015).

Uniendo el estudio de los roles profesionales con el de la sociología de las noticias, Mellado (2015) operacionaliza diferentes roles profesionales en el contenido noticioso según diferentes dominios de acción dentro del campo periodístico. Uno de dichos dominios se vincula a la forma en que el periodismo aborda a la audiencia, analizando si los periodistas utilizan estilos de reporteo que evocan más a un público como ciudadano, cliente o espectador.

Tal y como plantea Mellado (2015), la materialización de los roles profesionales en la práctica periodística (concepto acuñado como journalistic role performance) puede darse tanto al nivel del proceso de producción y de las rutinas periodísticas como en el producto informativo que el público lee, ve o escucha.

Siguiendo esta segunda vertiente de análisis, este artículo analizará la presencia de los roles profesionales de infoentretenimiento, cívico y de servicio en la cobertura de cultura y espectáculos dada por las noticias de televisión, 
radio, prensa escrita y online en Chile durante el primer semestre de 2015. El objetivo principal de este estudio es comprender la forma en que el periodismo aborda a la audiencia en la cobertura de cultura y espectáculos a través de distintos estilos de reporteo, y cómo dichas prácticas cambian o se mantienen estables en diversas plataformas informativas.

En Chile, los medios de comunicación se posicionan como la fuente primordial de acceso a la información y el entretenimiento (Labarca y Matta, 2010), por lo que tratar al público como espectador, ciudadano o cliente no es un tema trivial. En efecto, la forma en que el periodismo plantee al público puede hacer que los contenidos elegidos y la forma en que estos son planteados influyan en la misma concepción que el público tiene sobre lo que es, en este caso, la cultura y el espectáculo.

Según Güell y Peters (2010), la mayoría de los ciudadanos chilenos se informa de lo que ocurre por medio de la televisión, el que a su vez es el medio de comunicación más consumido por la población (Consejo Nacional de Televisión, 2013). Sin embargo, los sujetos adultos que declaran un alto consumo de noticias de cultura y espectáculos se informan mediante prensa escrita, y los jóvenes con estas mismas características, por internet.

Aunque las secciones dedicadas a la cultura y los espectáculos en las distintas plataformas de noticias existentes, usualmente, son consideradas temáticas "blandas” (Reinemann, Stanyer, Scherr y Legnante, 2012), entendiéndose estas como "asuntos que pueden ser considerados 'menos serios' y no relacionados a temas políticos, económicos y sociales de importancia” (Digón, 2005), los medios han comenzado a brindar mayor tiempo o espacio a estos contenidos (Burton, 2005; Uribe y Gunter, 2007).

\section{Roles profesionales y cómo el periodismo aborda a la audiencia}

La forma en que el periodismo aborda a la audiencia se vincula directamente con el debate entre el periodismo como servicio público y el periodismo como organización con fines meramente comerciales (Eide y Knight, 1999). 
Las distinciones clásicas cuando se analiza la forma en que los periodistas abordan a la audiencia corresponden a la dicotomía del público como ciudadano versus el público como consumidor (Reinemann, Stanyer, Scherr y Legnante, 2012). Sin embargo, diversos desarrollos teóricos desafían esta dicotomía proponiendo distintas tipologías (Eide y Knight, 1999; Atkinson, 2011; Costera Meijer, 2003). Según Mellado y Van Dalen (2017, p. 214), las transformaciones tecnológicas y sociales han modificado las formas de generar contenido por parte de los medios, siendo estas cada vez más híbridas, situacionales y complementarias. En este contexto, los periodistas han debido reconsiderar su relación con el público, ampliando sus estilos de reporteo y narrativas.

En su trabajo sobre roles profesionales y prácticas periodísticas, Mellado (2015) se basa en dicho desarrollo teórico y propone tres roles desde los cuales se puede abordar a la audiencia a través de la cobertura noticiosa de forma distinta pero al mismo tiempo complementaria: el rol de servicio, cuyo enfoque responde a la necesidad de informar acerca del acceso a bienes y servicios y donde se genera una relación cliente-profesional entre el público y el periodista; el rol cívico, relacionado con la responsabilidad social que tiene el periodismo con la vida pública y con un público como ciudadano; y el rol de infoentretenimiento, que busca entretener, informar y distraer a un público-espectador.

El rol cívico incorpora elementos del periodismo público (Rosen, 1996), en busca de motivar a la audiencia a que participe de la vida social, política y cultural, con fuertes rasgos de responsabilidad social (Merritt, 1998) y conciencia de vida pública, y se transforme en un puente entre las necesidades de la población y sus gobernantes, en tanto que el rol de servicio intenta colaborar con información y consejos sobre bienes y servicios a la audiencia que pueden resultar útiles para su vida diaria. Esta dimensión está relacionada con el estilo de vida de las personas en su rol de sujetos, dando solución a los problemas individuales que acontecen en la vida de estos (Eide y Knight, 1999; Mellado, 2015). Finalmente, el rol de infoentretenimiento aborda a la audiencia con contenidos más emocionales, a través de diferentes estilos narrativos o efectos visuales (Mellado, 2015). 
Mellado y Lagos (2014, p. 10) analizaron la presencia de estos tres roles en la cobertura que la prensa impresa chilena daba a las noticias vinculadas a la crónica nacional y encontró que el rol más presente en las noticias era el de infoentretenimiento, representado mayoritariamente a través del uso de emociones (14.3\%), sensacionalismo (13\%) y personalización (12\%).El rol cívico, se materializaba principalmente a través de la presencia de información de contexto $(11.8 \%)$, de la perspectiva de los ciudadanos (6.5\%), del impacto local (5.5\%) y de información sobre actividades ciudadanas (5\%). El rol de servicio, finalmente, estaba prácticamente ausente de la cobertura de la crónica nacional de la prensa impresa. Este estudio, sin embargo, solo analizaba la prensa impresa y noticias de la crónica nacional y dejaba de lado la diversidad que componen los sistemas de medios y las temáticas que ocupan parte importante de la agenda informativa, como lo son, entre otras, cultura y espectáculos.

\section{Roles profesionales en el contexto multiplataforma}

A lo largo de la historia, los cambios tecnológicos asociados al desarrollo de los medios han obligado a los periodistas a adaptar sus rutinas y sus prácticas de acuerdo con las necesidades de nuevas plataformas. Por ejemplo, la llegada de la radio cuando solo existía la prensa escrita introdujo elementos como la instantaneidad y reveló habilidades en los periodistas como su capacidad de improvisación. De la misma forma, la llegada de la televisión añadió aspectos visuales y otorgó importancia a la apariencia de estos y al lenguaje corporal que utilizan (Meltzer, 2010). La llegada de internet también generó cambios importantes en la forma de informar, la interacción con el público, la posibilidad de información 24/7 y los cambios en las rutinas y relación con las fuentes, así como el comienzo de la convergencia tecnológica de los medios, alterarían las rutinas periodísticas (Domingo et al., 2008). Por otro lado, junto con los cambios de los medios, surgen los cambios de las audiencias, las cuales requieren y exigen distintos tipos de contenido y una nueva forma de vincularse con la información periodística (Brants y De Haan, 2010). 
En este contexto, a lo largo de las décadas, se ha podido anticipar que la forma en que los medios se adaptan a estos cambios cobra suma relevancia en el debate público y privado (Maier, 2010).

Las investigaciones que se han propuesto la tarea de comparar el contenido noticioso simultáneamente entre distintas plataformas informativas son escasas (Reich, 2011), y hasta la fecha, no existen estudios conocidos que hayan analizado la materialización de los roles periodísticos en las noticias comparando más de una plataforma noticiosa.

Los estudios que han comparado más de una plataforma informativa dentro del campo de los medios de comunicación han medido otros aspectos de las noticias como las agendas noticiosas (Valenzuela y Arriagada, 2009) o rutinas periodísticas (Boczkowski y Ferris, 2005; Singer, 2004; Reich, 2011), entre otros.

La discusión en torno a las diferencias estructurales de las plataformas informativas, y el contenido noticioso que estas producen, se ha generado a partir de dos perspectivas teóricas (Reich, 2011). Por un lado, la perspectiva particularista, la cual postula que cada plataforma tiende a ejecutar determinadas prácticas profesionales por medio de culturas de producción específicas, determinadas por la línea editorial, rutinas periodísticas y estándares de cada medio (Deuze, 2008; Benson, Blach-Orsten, Powers, Willig y Zambrano, 2012).

Y por el otro lado, la perspectiva generalista, la cual postula que factores como los valores, la ideología profesional, así como la estandarización de diferentes procedimientos del trabajo periodístico, tienden a generar criterios comunes en la producción de noticias, sin importar la plataforma informativa (Reich, 2011). De acuerdo con esta perspectiva, la similitud entre medios también se debe por la convergencia tecnológica y organizacional (Cook, 1998; Boczkowski y Ferris, 2005), así como por estar insertos en un mismo contexto cultural (Bourdieu, 1998). 
Los estudios previos han aportado resultados que apoyan ambas perspectivas. Mientras algunos no han encontrado grandes diferencias en el contenido producido entre plataformas (Cohen, 2002; Druckman, 2005; Lee, 2007; Maier, 2010; Reich, 2011; Ghersetti, 2014), otros sí han encontrado diferencias significativas entre una plataforma y otra (Graber, 2003; Bennett, 2003; Curran, Iyengar, Lund y Salovaara-Moring, 2009; Benson, Blach-Orsten, Powers, Willig y Zambrano, 2012).

La evidencia muestra entonces resultados conflictivos a la hora de comparar aspectos informativos entre distintas plataformas y la inexistencia de estudios previos que hayan comparado roles profesionales en la cobertura de cultura y espectáculos en distintas plataformas simultáneamente.

\section{Cultura y espectáculos como frentes informativos}

No existe una delimitación explícita que defina dónde empieza el área de cultura y comienza el área de espectáculos (Meneses, 2007). Sin embargo, el establecimiento de áreas de cobertura existe dentro de los medios y permite que los periodistas se focalicen y logren cierta expertise, que se traduce en un tratamiento del contenido diferente del que podría otorgarle un periodista sin un frente temático definido (McCluskey, 2008).

Pese a que esta investigación tratará cultura y espectáculos como frentes temáticos diferentes, no siempre existe una distinción explícita entre una sección y otra en las distintas plataformas mediáticas existentes. Incluso entre medios del mismo tipo, la conceptualización de uno y otro frente temático varía bastante. Uno de los fenómenos a los que se ha vinculado esta situación es la llamada tabloidización de las noticias, la cual "describe el cambio de enfoque de las noticias más 'duras' a lo salaz, al sensacionalismo y al entretenimiento" (Alotaibi, 2013, p. 1), en busca de lograr mayor interés de las audiencias, mediante la exaltación de algunos atributos, como la vida privada (Blumler y Gurevitch, 1995; Uribe y Gunter, 2007).

En efecto, de acuerdo con estudios precedentes, las funciones de entretener, educar a la ciudadanía sobre asuntos púbicos o ayudar al público en sus problemas cotidianos pueden estar presentes tanto en cultura y es- 
pectáculos como en frentes más duros, como política, nacional o economía (Uribe y Gunter, 2007; Mellado y Lagos, 2014; Wang, Sparks, Lü y Huang, 2017; Mellado, López y Elórtegui, 2017), apoyando la idea de que los periodistas de distintos frentes informativos pueden llevar a cabo diferentes roles simultáneamente.

Aunque los estudios sobre el incremento de la tabloidización y el infotainment son considerables, estos se enfocan principalmente en cómo las noticias de política u otros frentes se han espectacularizado, sobre todo en televisión (Blumler y Gurevitch, 1995; Uribe y Gunter, 2007). Por el contrario, no es posible encontrar análisis de contenidos de las secciones de espectáculos de forma específica, pero sí ensayos que definen y discuten en torno al término.

Catalogar la prensa de cultura y espectáculos como prensa blanda por los contenidos que trata no es algo que pueda darse por sentado, en tiempos cuando la tabloidización ha permitido que contenidos duros, como la política y la economía, sean presentados más livianamente de acuerdo con la forma de ser abordados (Uribe y Gunter, 2007; Mellado y Van Dalen, 2017). Esto ha permitido que las divisiones entre prensa dura y blanda sean difusas, y que la unidad noticiosa sea un producto híbrido (Mellado, 2015).

En cuanto a la "tabloidización" del espectáculo como frente noticioso en Chile, Barrientos (2010) plantea su origen en 1999, con un episodio en el que la prensa se percató de la popularidad que alcanzaban estas noticias. Dos años después, en 2001, saldría al aire el primer programa de televisión dedicado exclusivamente a la farándula (Primer Plano de CHV); y en 2002, el diario Las Últimas Noticias daría un vuelco en su línea editorial "pasando de la crónica roja al espectáculo" (Barrientos, 2010, p. 54).

Aunque la discusión en torno a lo que es el periodismo de cultura y el periodismo de espectáculos no ha logrado un consenso teórico, ni tampoco una consenso sobre la forma de operacionalizar ambos tipos de cobertura (Villa, 2000; Rubiano, 2006). Esta investigación se sustentará en el planteamiento de García Canclini (1990), quien habla de un encuentro 
entre la tradición popular, lo culto y lo masivo a la hora de referirnos a estas temáticas, y donde una expresión artística puede responder a más de una de estas dimensiones. Esta visión se ajusta a la práctica periodística chilena, en la que los límites entre periodismo cultural y de espectáculos no están consensuados, y varían de acuerdo con la plataforma informativa y el medio específico que se analiza.

Según la discusión de la literatura previa, a continuación planteamos las preguntas formales de investigación que guiarán nuestro estudio:

P.1. ¿Existen diferencias significativas en la presencia de los roles profesionales de infoentretenimiento, cívico y de servicio entre las noticias de cultura y de espectáculo en el periodismo chileno?

P.2. ¿Existen diferencias significativas en la presencia de los roles profesionales de infoentretenimiento, cívico y de servicio en la cobertura de noticias de cultura y espectáculos dependiendo de la plataforma informativa?

\section{Metodología}

El diseño metodológico de esta investigación se basa en un estudio cuantitativo, específicamente en un análisis de contenido de las noticias de prensa impresa, televisión, radio y prensa online chilena durante el primer semestre de 2015.

\section{Muestra}

Para obtener una muestra comparable y válida, se siguió un protocolo común. Dentro de cada medio, las unidades de muestreo correspondieron a los ejemplares, home page, ediciones y noticieros seleccionados, y la unidad de análisis fue el ítem noticioso. La elección de las unidades de muestreo se determinó a partir de los niveles de audiencia y lectoría de cada medio.

En TV se analizaron los noticieros centrales de TVN y Chilevisión. En radio, los programas centrales de radio Biobío y Cooperativa. En prensa escrita, se analizaron La Tercera y Las Últimas Noticias, ambos de circu- 
lación nacional y representantes de la prensa élite y popular. En medios online, los portales El Mostrador y El Dínamo.

Por medio del método de la semana construida (Riff, Lacy y Fico, 2014, pp. 112-117), se seleccionó una muestra estratificado-sistemática de cada uno de los medios. En cada uno de ellos, se escogió aleatoriamente un lunes, martes, miércoles, jueves, viernes, sábado y domingo de cada uno de los meses de la muestra, asegurándonos de que cada mes del año estuviera representado por al menos un día, y evitar la sobrerrepresentación de un mes en particular. Dentro de cada ejemplar, edición, noticieros y publicaciones seleccionadas, se analizaron todas las piezas informativas (noticias) publicadas o emitidas en dichos espacios vinculados a la cobertura de cultura y espectáculos.

Se excluyeron, en todas las plataformas, los bloques, los espacios o las secciones de editorial, columnas de opinión, tiempo, horóscopo, cartelera, juegos y vida social. También se excluyeron los suplementos, las revistas y los programas de reportajes especiales publicados o emitidos por los medios de la muestra. En los diarios, tampoco se incluyeron los anuncios de portadas, y en la TV y radio, los titulares y llamados. En el caso de la prensa escrita, se analizaron los cuerpos del diario que aparecen todos los días, mientras que en la prensa online se estudió el primer enlace que aparecía en el portal web.

Se consideraron noticias de espectáculos todas las noticias referidas a actividades que involucran personajes o celebridades del mundo del entretenimiento o del espectáculo y aquellas que cubren eventos populares de audiencia masiva, tales como shows, festivales, actividades desarrolladas por personajes de la televisión, parejas famosas, chismes, etc. En tanto que se consideraron noticias de cultura todos los eventos, las situaciones y las actividades relacionadas con expresiones artísticas (literatura, cine, danza, música clásica, etc.), históricas, culinarias y académicas, vinculadas a actividades culturales orientadas a una audiencia de élite (campo de producción restringida). Esta categoría incluye noticias sobre libros, discos, música, lanzamiento de películas, entre otros. También expresiones de grupos 
o colectivos sociales. En total, se encontraron 237 ítems noticiosos vinculados a estas temáticas dentro de las unidades de muestreo seleccionadas.

\section{Medidas}

Para medir la forma en que el periodismo de cultura y espectáculo aborda a la audiencia, se utilizó la instrumentalización propuesta por Mellado (2015) y validada por Mellado y Van Dalen (2017) en la prensa chilena, así como diferentes estudios internacionales antes mencionados. Específicamente, los roles cívico, de servicio y de infoentretenimiento fueron examinados a través de la presencia o ausencia de indicadores específicos en el contenido noticioso.

El rol de servicio fue medido con cinco indicadores: impacto en la vida diaria, consejos (quejas) y consejos (riesgos individuales), información de consumo y consejos de consumo. El rol de infoentretenimiento fue medido con seis indicadores: 1) personalización, 2) vida privada, 3) sensacionalismo, 4) escándalo, 5) emociones y 6) morbo. Finalmente, el rol cívico fue medido a través de nueve indicadores: 1) perspectiva ciudadana, 2) demanda ciudadana, 3) credibilidad de los ciudadanos, 4) impacto local y en la comunidad, 5) educación en deberes y derechos, 6) información de contexto, 7) preguntas de los ciudadanos, 8) información sobre las actividades de los ciudadanos y 9) apoyo a los movimientos ciudadanos.

Para fines descriptivos, se reporta el promedio simple (la suma de los puntos dividida por el total de ítems en cada rol) y la desviación estándar de la puntuación de cada rol, así como los porcentajes de los indicadores que los componen. Para calcular las diferencias entre grupos a través de t-tests o pruebas de ANOVA, se utilizó la puntuación factorial (factor scores).

\section{Recolección de datos y codificación}

Para recolectar la muestra correspondiente a prensa escrita, se procedió a adquirir ejemplares de los periódicos seleccionados, grabar los noticieros de televisión, descargar las noticias presentes en el home page de la prensa digital del día seleccionado y grabar los noticieros centrales de la radio. 
El análisis de las unidades noticiosas fue codificado por dos personas capacitadas para dichos efectos. Según la fórmula de alfa de Krippendorf, la confiabilidad interjueces global fue de 0.78 .

\section{Resultados}

En relación con las noticias de cultura, en términos generales, los resultados muestran un predominio igualitario de los roles de infoentretenimiento $(\mathrm{M}=0.24 ; \mathrm{DS}=0.18)$ y de servicio $(\mathrm{M}=0.24 ; \mathrm{DS}=0.18)$ en las noticias de cultura en Chile. Mucho más lejana se observa la presencia del rol cívico $(M=0.05 ; \mathrm{DS}=0.13)$.

$\mathrm{Al}$ analizar los indicadores que integran cada rol por separado, es posible apreciar el predominio de algunos por sobre otros, dentro de un mismo rol (tabla 1 ).

\section{Tabla 1. Concepción de audiencia en el periodismo de cultura y espectáculos}

\begin{tabular}{|c|c|c|c|c|c|c|}
\hline & \multicolumn{2}{|c|}{ Servicio } & \multicolumn{2}{|c|}{ Infoentretenimiento } & \multicolumn{2}{|c|}{ Cívico } \\
\hline & Cultura (\%) & $\begin{array}{c}\text { Espectáculos } \\
(\%)\end{array}$ & Cultura (\%) & $\begin{array}{c}\text { Espectáculos } \\
(\%)\end{array}$ & Cultura (\%) & $\begin{array}{c}\text { Espectáculos } \\
(\%)\end{array}$ \\
\hline $\begin{array}{c}\text { Impacto en la vida } \\
\text { diaria }\end{array}$ & 4.8 & 0 & & & & \\
\hline Consejo 1 & 0 & 1.1 & & & & \\
\hline Consejo 2 & 0 & 3.4 & & & & \\
\hline $\begin{array}{c}\text { Información de } \\
\text { consumo }\end{array}$ & 67.7 & 32 & & & & \\
\hline Consejos de consumo & 46.8 & 19.4 & & & & \\
\hline Personalización & & & 67.7 & 62.9 & & \\
\hline Vida privada & & & 16.1 & 24.6 & & \\
\hline Sensacionalismo & & & 21 & 37.7 & & \\
\hline Emociones & & & 29 & 40 & & \\
\hline Morbo & & & 8.1 & 4.6 & & \\
\hline Escándalo & & & 0 & 0.6 & & \\
\hline Perspectiva ciudadana & & & & & 9.7 & 2.9 \\
\hline Demanda ciudadana & & & & & 3.2 & 0 \\
\hline Credibilidad ciudadana & & & & & 9.7 & 2.9 \\
\hline $\begin{array}{l}\text { Impacto local y en la } \\
\text { comunidad }\end{array}$ & & & & 11.3 & 4 & \\
\hline
\end{tabular}




\begin{tabular}{|c|l|l|l|l|l|l|}
\hline $\begin{array}{c}\text { Educación en deberes y } \\
\text { en derechos }\end{array}$ & & & & 1.6 & 1.1 & \\
\hline $\begin{array}{c}\text { Información de } \\
\text { contexto }\end{array}$ & & & & 0 & 11.3 & 2.9 \\
\hline $\begin{array}{c}\text { Preguntas de los } \\
\text { ciudadanos }\end{array}$ & & & 3.2 & 2.3 & \\
\hline $\begin{array}{c}\text { Información sobre } \\
\text { actividades ciudadanas }\end{array}$ & & & & 0 & 0.6 & \\
\hline $\begin{array}{c}\text { Apoyo a movimientos } \\
\text { ciudadanos }\end{array}$ & & & 0.6 & \\
\hline
\end{tabular}

Fuente: elaboración propia.

En el rol de servicio, la entrega de información de consumo y los consejos de consumo tienen un protagonismo bastante mayor. Dentro del rol de infoentretenimiento, se observa una mayor presencia de la personalización, seguido más atrás de emociones y sensacionalismo. En cuanto al rol cívico, aunque menos presente, tienen mayor presencia el impacto local y en la comunidad, la información de contexto, la perspectiva ciudadana y la credibilidad ciudadana.

Paralelamente, en el caso de las noticias de espectáculos, los datos muestran que el rol más predominante es el de infoentretenimiento $(\mathrm{M}=0.28 ; \mathrm{DS}=0.20)$, seguido del de servicio $(\mathrm{M}=0.11 ; \mathrm{DS}=0.16)$. La presencia del rol cívico, en cambio, es casi nula cuando se analizan los datos agregadamente $(\mathrm{M}=0.01 ; \mathrm{DS}=0.08)$.

Dentro del rol de infoentretenimiento prima la personalización, seguida de las emociones y el sensacionalismo en las noticias sobre espectáculos. En tanto que, y nuevamente, el rol de servicio alcanza mayor presencia a través de información de consumo y consejos de consumo.

Cuando se compara la presencia de estos roles entre ambas temáticas, los resultados muestran diferencias significativas en la presencia del rol de servicio $(\mathrm{t}=4.999 ; \mathrm{gl}=235 ; \mathrm{p}=0.000)$ y del rol cívico $(\mathrm{t}=2.432$; $\mathrm{gl}=235 ; \mathrm{p}=0.02$ ), pero no así en la presencia del rol de infoentretenimiento $(\mathrm{t}=-1.596 ; \mathrm{gl}=235 ; \mathrm{p}=0.11)$ entre las noticias de cultura y espectáculos. El tamaño del efecto $\left(\eta^{2}=0.18\right)$ es alto en el caso del rol cívico, y pequeño, pero aún relevante, en el caso del periodismo de servicio $\left(\eta^{2}=0.02\right)$. 
En el caso del rol de servicio, este tiene significativamente mayor presencia en las noticias de cultura $(M=0.24 ; D S=0.18)$ que en las noticias de espectáculos $(\mathrm{M}=0.11$; $\mathrm{DS}=0.17)$, tal como sucede en el caso del rol cívico. En el periodismo de infoentretenimiento, en cambio, aunque existe mayor presencia en la cobertura de espectáculos $(M=0.28 ; D S=0.20)$, que en la de cultura $(M=0.24 ; D S=0.18)$, las diferencias no son significativas.

\section{Rol de servicio, infoentretenimiento y cívico según plataforma informativa}

Para reportar los resultados de este apartado, se analizaron los datos de cultura y espectáculos de forma agregada. Los resultados muestran diferencias significativas en la presencia del rol de servicio $(\mathrm{F}=3.883 ; \mathrm{gl}=3 ; \mathrm{p}=0.010$; $\left.\eta^{2}=0.05\right)$, de infoentretenimiento $\left(\mathrm{F}=6.973 ; \mathrm{gl}=3 ; \mathrm{p}=0.000 ; \eta^{2}=0.08\right)$ y del rol cívico $\left(\mathrm{F}=33.458 ; \mathrm{gl}=3 ; \mathrm{p}=0.000 ; \eta^{2}=0.03\right)$ en la cobertura de noticias de cultura y espectáculos dependiendo de la plataforma informativa. El tamaño del efecto $\left(\eta^{2}\right)$ es alto en el caso del rol de infoentretenimiento, y solo moderado, pero aún importante, en los otros dos roles.

Como se puede apreciar en la tabla 2, en el caso del rol de servicio, las diferencias se dan entre la prensa y los medios online, estando este rol significativamente más presente en la prensa digital que en los diarios impresos. En la TV y la radio, la presencia del periodismo de servicio es similar en la cobertura de ambos tópicos.

Tabla 2. Presencia de los roles de servicio, infoentretenimiento y cívico en la cobertura de cultura y espectáculos según plataforma informativa (media y desviación estándar)

\begin{tabular}{|c|c|c|c|c|}
\hline & Prensa impresa & Prensa digital & TV & Radio \\
\hline Rol de servicio & $0.12(0.17)$ & $0.22(0.19)$ & $0.18(0.21)$ & $0.19(0.19)$ \\
\hline Rol de infoentrenimiento & $0.26(0.20)$ & $0.22(0.18)$ & $0.44(0.21)$ & $0.25(0.15)$ \\
\hline Rol cívico & $0.00(0.03)$ & $0.02(0.07)$ & $0.20(0.24)$ & $0.03(0.08)$ \\
\hline
\end{tabular}

Fuente: elaboración propia. 
En el caso del rol de infoentretenimiento, este está significativamente más presente en las noticias de televisión que en cualquiera de las otras plataformas analizadas. Cabe destacar que la media del rol de infoentretenimiento en las noticias televisivas dobla la media de su presencia en los medios digitales, cuya existencia es la más baja entre los cuatro tipos de medios analizados.

Finalmente, las diferencias en la presencia del rol cívico en la cobertura noticiosa se dan entre la televisión y las otras tres plataformas analizadas, con la televisión mostrando una mayor presencia de este rol en su cobertura noticiosa de cultura y espectáculos.

\section{Discusión y conclusiones}

Por medio de un análisis de contenido de noticias de cultura y de espectáculos, este estudio analizó la forma en que el periodismo de televisión, radio, prensa escrita y online chileno aborda a la audiencia a través de la materialización de los roles profesionales cívico, de servicio e infoentretenimiento en ambos frentes noticiosos.

En términos agregados, el infoentretenimiento es un rol predominante y transversal a la cultura y el espectáculo. En efecto, es el rol más presente en ambas temáticas, sin observarse diferencias significativas en su presencia entre estos dos frentes informativos.

Por su parte, el rol de servicio está altamente presente en la cobertura cultural a la par que el infoentretenimiento, mientras que el desarrollo del rol cívico por parte de los periodistas no es prioridad en las noticias de ninguno estos frentes, aunque la televisión adelanta significativamente a las demás plataformas en su presencia.

Que los periodistas de cultura consideren a sus audiencias como espectadores y clientes, por sobre su condición de ciudadanos, resulta revelador si se toma en cuenta la función social que cumple la profesión periodística en la difusión de la cultura, el patrimonio y las artes. Considerando la influencia que el periodismo y los medios tienen en la construc- 
ción de realidad a la hora de generar, organizar y jerarquizar el contenido noticioso (McCombs, 2004), estos resultados tienden a sustentar la idea que los medios y el periodismo estarían supeditados a la generación de entretención y de necesidades de consumo de las personas, más que a la creación de una identidad cultural y ciudadana desde estos frentes.

En la cobertura de espectáculos, se observa una mayor presencia del rol de infoentretenimiento, seguido del rol de servicio, y muy lejanamente del periodismo cívico. Esto indica que los medios dirigen la cobertura de espectáculos a un público considerado más como espectador, y en alguna medida cliente, que como ciudadano.

La alta presencia de infoentretenimiento, tanto en las noticias de cultura como de espectáculos, es concordante con estudios previos sobre roles profesionales en noticias duras como política, economía y negocios, nacional, policía y tribunales y asuntos sociales (Mellado y Lagos, 2014), donde se identificó al infoentretenimiento como el segundo rol más presente en la prensa escrita nacional. En este sentido, estos resultados tienden a contradecir la idea, así como lo han hecho estudios precedentes, que las noticias con temáticas más "blandas" poseen mayor infoentretenimiento que las noticias con temáticas más "duras".

Aunque el afán de servicio de la cultura y los espectáculos resulte coherente con la cercanía de ambas temáticas a informar sobre eventos y productos culturales y artísticos, se debe tener en cuenta el papel que asume el periodismo al establecer una agenda cultural y de espectáculos que da prioridad a determinadas temáticas o áreas, como las películas, la música y la televisión, por sobre otras áreas, como la danza, las artes visuales o el teatro.

En efecto, los análisis posteriores efectuados sobre los datos aquí reportados muestran que la presencia del rol de infoentretenimiento no depende del tipo de subtópico presente en la cobertura de cultura y de espectáculos; por ejemplo, aunque una noticia trate una subtemática asociada a la cultura de élite, como lo es el ballet, esta subtemática tiene tantas posibilidades de incluir infoentretenimiento como lo tendría una noticia sobre el divorcio de un actor famoso. 
Aunque los contenidos planteados por las temáticas de cultura y espectáculos en Chile varían de acuerdo con cada medio y plataforma, existe una tendencia común de los medios de diferenciar ambos frentes según cuán masivo o de élite sea el contenido. A diferencia quizá de otras culturas periodísticas, ambos temas son claramente separados dentro de las salas de redacción. No obstante, se puede asumir que los estilos de reporteo entre el periodismo de cultura y de espectáculos, si bien varían, no lo hacen de una forma radical.

En cuanto a las diferencias en la materialización de los roles profesionales en las distintas plataformas informativas, aunque el infoentretenimiento es el rol que más predomina en las cuatro plataformas analizadas, es en la televisión donde la presencia de este estilo de reporteo es más predominante. Del mismo modo, la televisión es el medio donde más predominio tiene el periodismo cívico.

Una posible explicación de esta realidad es que en la televisión aún existe la práctica de consultar a los ciudadanos acerca de sus opiniones respecto de noticias o sucesos de actualidad, algo que puede lograr mayor credibilidad que en otros medios, ya que las audiencias pueden ver el rostro, oír la voz o leer el nombre de la fuente ciudadana en el texto o Generador de Caracteres utilizado por la televisión (GC). Sin embargo, también podría suceder que la televisión genere un efecto de seudociudadanía, al utilizar ciudadanos ordinarios para ejemplificar una historia, más que para darles legítima voz y empoderarlos.

La mayor presencia del rol de infoentretenimiento en televisión, en comparación con las demás plataformas, puede tener que ver también con la relación del infoentretenimiento de los elementos audiovisuales propios de esta plataforma que hace más fácil que este rol se desarrolle. En televisión, la personalización y las emociones desempeñan un papel clave en cuanto la plataforma audiovisual permite encuadres más dinámicos.

En tal sentido, los hallazgos de este estudio tienden a apoyar más bien la perspectiva particularista, en relación con la forma en que el perio- 
dismo de cultura y espectáculos aborda a la audiencia en distintas plataformas noticiosas.

A pesar del análisis de roles profesionales y su materialización en el periodismo de cultura y espectáculos chileno, aportados por este estudio, existen limitaciones considerables por mencionar. Una de las más importantes es que, por la naturaleza de esta investigación, no se analizaron columnas de opinión o críticas especializadas, pese al fuerte predominio de este formato en las secciones de cultura y de espectáculos. Por otra parte, tampoco se estudiaron noticias presentes en programas o suplementos dedicados exclusivamente a estas temáticas. Es también de esperar, más en el plano comparado, que futuros estudios comparen el periodismo de cultura y de espectáculo en Chile con el de otros países y regiones.

\section{Referencias}

Alotaibi, N. (2013). An analysis of the implications of the growing tabloidization of news coverage on democratic politics. European Scientific Journal, 9(14), 185-195.

Atkinson, J. (2011). Performance journalism: A three-template model of television news. The International Journal of Press/Politics, 16(1), 102-129.

Barrientos Muñoz, E. M. (2010). Periodismo de farándula en Chile: consolidación de de un fenómeno (Tesis de grado, Universidad de Chile, Santiago de Chile).

Bennett, W. (2003). News: The politics of illusion. Nueva York: Longman.

Benson, R., Blach-Orsten, M., Powers, M., Willig, I. y Zambrano, S. (2012). Media systems online and off: Comparing the form of news in the United States, Denmark and France. Journal of Communication, 62(1), 21-38. 
Blumler, J. y Gurevitch, M. (1995). The crisis of public communication. Nueva York: Routledge.

Boczkowski, P.J. y Ferris, J. A. (2005). Multiple media, convergent processes, and divergent products: Organizational innovation in digital media production at a European firm. The Annals of the American Academy of Political and Social Science, 597(1), 32-47.

Bourdieu, P. (1998). On television. Nueva York: New Press.

Brants, K. y De Haan, Y. (2010). Taking the public seriously: Three models of responsiveness in media and journalism. Media, Culture \& Society, 32, 411-428.

Burton, G. (2005). Media and society: Critical perspectives. Berkshire: McGraw-Hill Education.

Cohen, E. L. (2002). Online journalism as market-driven journalism. Journal of Broadcasting Electronic Media, 46(4), 532-548.

Consejo Nacional de Televisión (2013). La importancia de los noticiarios televisivos para las familias chilenas. Recuperado de https://goo. $\mathrm{gl} /$ AsvgXi

Cook, T. (1998). Governing with the news: The news media as a political institution. Chicago: University of Chicago Press.

Costera Meijer, I. (2003). What is quality television news? A plea for extending the professional repertoire of newsmakers. Journalism Studies, 4, 15-29.

Curran, J., Iyengar, S., Lund, A.B. y Salovaara-Moring, I. (2009). Media system, public knowledge and democracy: A comparative study. European Journal of Communication, 24(1), 5-26. 
Deuze, M. (2008). The professional identity of journalists in the context of convergence culture. Observatorio, 2(4), 103-117.

Digón Regueiro, P. (2005). Las “otras noticias” y los telediarios españoles: una propuesta de análisis. Comunicar, 25, 1-11.

Domingo, D., Quandt, T., Heinonen, A., Paulussen, S., Singer, J.y Vujnovic, M. (2008). Participatory journalism practices in the media and beyond: An international comparative study of initiatives in online newspaper. Journalism Practice, 2(3), 326-342.

Druckman, J. (2005). Media matter: How newspapers and television news cover campaigns and influence voters. Political Communication, $22(4), 463-481$.

Eide, M. y Knight, G. (1999). Public/private service: Service journalism and the problems of everyday life. European Journal of Communication, 14(4), 525-547.

García Canclini, N. (1990). Culturas híbridas. México: Grijalbo.

Ghersetti, M. (2014). Still the same? Comparing news content in online and print media. Journalism Practice, 8(4), 373-389.

Graber, D. (2003). The media and democracy: Beyond myths and stereotypes. Annual Review of Political Science, 6, 139-160.

Güell, P. y Peters, T. (2010). Las mediaciones de la cultura: ¿qué medios de información utilizan los chilenos para informarse de la oferta de bienes y servicios culturales? RE-Presentaciones, 3(6), 43-60.

Labarca, B. y Matta, C. (2010). Radiografía al sistema chileno de medios. Santiago: LOM. 
Lee, J. K. (2007). The effect of the Internet on homogeneity of the media agenda: A test of the fragmentation thesis. Journalism \& Mass Communication Quarterly, 84(4), 745-760.

Maier, S. (2010). All the news fit to post? Comparing news content on the web to newspapers, television, and radio. Journalism \& Mass Communication Quarterly, 87(3-4), 548-562.

McCluskey, M. (2008). Reporter beat and content differences in environmental stories. Journalism \& Mass Communication Quarterly, 85(1), 83-98.

McCombs, M. (2004). Setting the agenda: The mass media and public opinion. Cambridge, UK: Polity Press.

Mellado, C. (2015). Professional roles in news content: Six dimensions of journalistic role performance. Journalism Studies, 16(4), 596-614.

Mellado C., Hellmueller, L. y Donsbach, W. (2017). Journalistic role performance: Concepts, models, and measures. Nueva York: Routledge.

Mellado, C. y Lagos, C. (2014). Professional roles in news content: Analyzing journalistic performance in the Chilean national press. International Journal of Communication, 8, 2090-2112.

Mellado, C. y Van Dalen, A. (2014). Between rhetoric and practice. Journalism Studies, 15(6), 859-878.

Mellado, C. y Van Dalen, A. (2017). Challenging the citizen-consumer dichotomy: A news content analysis of audience approaches in Chilean Journalism. Journalism \& Mass Communication Quarterly, 94(7), 213-237.

Mellado, C., López-Rabadán, P. y Elortegui, C. (2016). ¿ (Para) ciudadanos o espectadores? Periodismo político chileno y sus modelos profesionales de aproximación a la audiencia. Palabra Clave, 20(1), 14-46. 
Meneses, M. (2007). En torno al periodismo especializado: consensos y disensos conceptuales. Análisis, 35, 137-152.

Meltzer, K. (2010). TV news anchors and journalistic tradition: How journalists adapt to technology. Nueva York: Peter Lang.

Merritt, D. (1998). Public journalism and public life: Why telling the news is not enough. Nueva Jersey: Lawrence Erlbaum.

Reich, Z. (2011). Comparing reporters' work across print, radio, and online: Converged origination, diverged packaging. Journalism \& Mass Communication Quarterly, 88(2), 285-300.

Reinemann, C, Stanyer, J., Scherr, S. y Legnante, G. (2012). Hard and soft news: A review of concepts, operationalizations and key findings. Journalism, 13(2), 221-239.

Riff, D., Lacy, S. y Fico, F. (2014). Analyzing media messages: Using quantitative content analysis in research. Mahwah, NJ: Erlbaum.

Rubiano, E. (2006). Escenario, butaca y ticket: el mercado de la cultura en el periodismo cultural. Tabula Rasa, 5, 129-148.

Rosen, J. (1996). Getting the connections right: Public journalism and the troubles in the press. Nueva York: Twentieth Century Fund Press.

Singer, J. (2004). More than inkstained wretches: The resocialization of print journalists in converged newsrooms. Journalism \& Mass Communication Quarterly, 81(4), 838-856.

Tandoc, E., Hellmueller, L. y Vos, T. (2013). Mind the gap: Between role conception and role enactment. Journalism Practice, 7(5), 539-554.

Uribe, R. y Guter, B. (2007). Are 'sensational' news stories more likely to trigger viewers' emotions than non-sensational news stories? A 
content analysis of British TV news. European Journal of Communication, 22(2), 207-228.

Valenzuela, S. y Arriagada, A. (2009). Competencia por la uniformidad en noticieros y diarios chilenos 2000-2005. Cuadernos de Información, 24(1), 43-45.

Villa, M. J. (2000). Una aproximación teórica al periodismo cultural. Revista Latina de Comunicación Social, 3(35), 1-17.

Vos, T. (2002). Role enactment: The influence of journalists' role conceptions on news content. Ponencia presentada en 85th AEJMC Conference, Miami, Florida.

Vos, T. (2005). Journalistic role conception: A bridge between the reporter and the press. Ponencia presentada en International Communication Association (ICA) conference, Journalism Studies Division, Nueva York, NY.

Wang, H., Sparks, C., Lü, N. y Huang, Y. (2017). Differences within the mainland chinese press: A quantitative analysis. Asian Journal of Communication, 27(2), 154-171. 